\title{
High angular resolution observations in the near infrared and modeling of the peculiar envelope of HD 62623
}

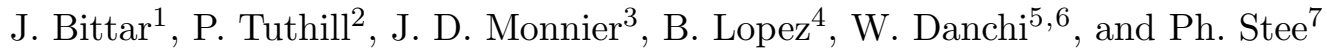 \\ 1 Observatoire Midi-Pyrénées, CNRS UMR 5572, 14 Av. Edouard Belin, 31400 Toulouse, France \\ 2 Chatterton Astronomy Departement, School of Physics, University of Sydney, NSW 2006, Australia \\ 3 Center for Astrophysics, 60 Garden Street, Cambridge, MA 02138, USA \\ 4 Observatoire de la Côte d'Azur, Département Fresnel UMR 6528, BP 4229, 06034 Nice Cedex 4, France \\ 5 NASA Goddard Space Flight Center, Infrared Astrophysics Branch, Code 685, Greenbelt, MD 207741, USA \\ 6 Space Sciences Laboratory, University of California at Berkeley, Berkeley, CA 94720-7450, USA \\ 7 Observatoire de la Côte d'Azur, Département Fresnel UMR 6528, Caussols, 06460 St. Vallier de Thiey, France
}

Received 20 June 2000 / Accepted 19 December 2000

\begin{abstract}
We report new observations of the peculiar star HD 62623 obtained with aperture masking interferometry performed on the Keck I telescope at $\lambda=1.24,1.65,2.26$ and $3.08 \mu \mathrm{m}$. The envelope around this star appears partially resolved in the near infrared except at $1.65 \mu \mathrm{m}$. Radiative transfer modeling of the dust shell of this star has been performed in spherical geometry. This modeling has two goals: it provides a framework for understanding the partially resolved object shown by the visibility curves, and it allows investigation of the envelope of HD 62623 by discrimination between proposed models. We show that reasonable fits to photometric observations can be obtained with a simple spherically symmetric silicate dust shell. Nevertheless, our high angular resolution measurements bring some important constraints on the modeling of the circumstellar environment of HD 62623. More realistic model involving the presence of a non spherically distributed dust envelope including the gas component in the circumstellar environment is required.
\end{abstract}

Key words. circumstellar matter - stars: individual (HD 62623) - techniques: interferometric

\section{Introduction}

The peculiar bright star HD 62623 (also named 3 Puppis) is classified as an A2Iabe supergiant in the Yale Bright Star Catalogue (Hoffleit \& Jaschek 1982). The distance of the object is estimated to be $d=700 \pm 150$ pc. Trams et al. (1990) proposed that HD 62623 is a massive star with a low mass unseen companion. A disk of gas and dust surrounds the whole system.

The binary nature is deduced from the radial velocity which varies with a period of 137.7 days (Lambert 1988). HD 62623 seems to be a system with a massive primary of radius $R_{\star}=55 \pm 10 R_{\odot}$ and a small mass ratio of $0.03<M 2 / M 1<0.15$, while $a_{1} \sin i$ is $6.5310^{6} \mathrm{~km}$ with $a_{1}$ the semi-major axis of the orbit of the primary.

An Infrared excess (Allen 1973; Trams et al. 1991) producing continuum emission between $\lambda=1 \mu \mathrm{m}$ and $\lambda=100 \mu \mathrm{m}$, was found by Rovero \& Ringuelet (1994) to be due to free-free and free-bound emission from the

Send offprint requests to: J. Bittar, e-mail: bittar@obs-mip.fr circumstellar material. No dust was required in their interpretation.

Allen (1973) found that the colour indices ( $H-K$ and $K-L$ ) for HD 62623 are too large to be due to freefree radiation. He proposed a competing model in which the IR excess can be accounted for by re-radiation from circumstellar dust clouds. Plets et al. (1995) also interpreted the significant IR excess observed for HD 62623 as due to the presence of circumstellar dust in a shell that extends from 35 to 5000 stellar radii. In this model the density falls as $R^{-1.3}$. This was deduced from the Infrared Astronomical Satellite (IRAS) Low-Resolution Spectrograph (LRS) spectrum which distinctly shows the $9.7 \mu \mathrm{m}$ silicate feature typical for O-rich stars. They interpreted the emission lines observed for HD 62623 as originating in a disk of gas surrounding the binary system.

This paper presents some new constraints brought by high angular resolution observations for HD 62623 obtained at the Keck I telescope in December 1997 and 


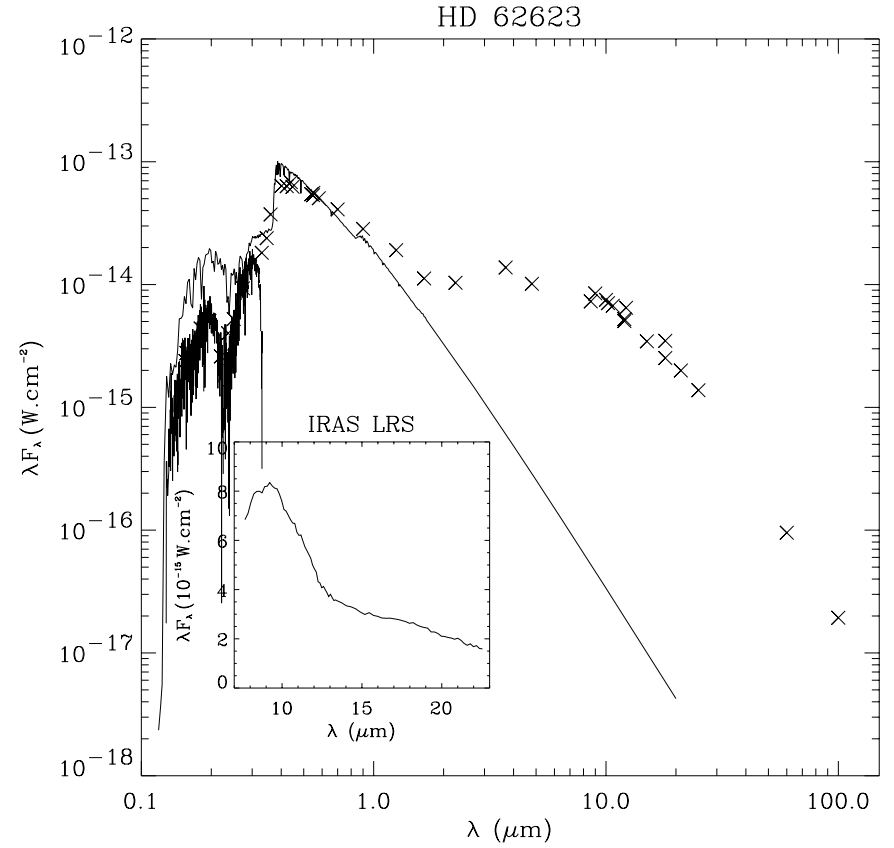

Fig. 1. The spectral energy distribution of the star HD 62623 where the flux $\lambda F_{\lambda}\left(\mathrm{Wcm}^{-2}\right)$ is plotted vs. $\lambda(\mu \mathrm{m})$. Data points (crosses) are taken from Table 1. The spectrum for $\lambda<0.33 \mu \mathrm{m}$ is from the IUE satellite, while the solid line is a solar composition Kurucz (1979) model atmosphere. The inset shows the low resolution spectrum of IRAS. Photometric observations are taken from Trams et al. (1991). No extinction correction was applied to the Kurucz model

April 1999. Radiative transfer modeling of the dust shell of this star is performed in spherical geometry. This modeling has two goals: to provide some confidence to the very partially resolved object and also to explain, by testing and excluding some possible models, the circumstellar envelope of HD 62623 and its IR excess. In Sect. 2 we compile the photometric data which exists in the literature then describe and show the Keck I observations. The modeling of HD 62623 assuming a spherical dusty envelope with no gas component is presented in Sect. 3. In Sect. 4 we highlight the new results and their limitations, and summarize the conclusions of this work.

\section{Observations}

\subsection{Photometric data}

Photometric data of HD 62623 available in the literature covers the whole energy distribution from the UV to the far IR. Photometric observations, taken from Rovero \& Ringuelet (1994) and from Plets et al. (1991), are listed in Table 1. The ultraviolet spectra are taken from the International Ultraviolet Explorer (IUE) observations. A low resolution spectrum for HD 62623 between 7.7 and $22.6 \mu \mathrm{m}$ is taken from the IRAS catalogue. The spectra are in two parts, one ranging from 7.7 to $13.4 \mu \mathrm{m}$ and the other from 11.0 to $22.6 \mu \mathrm{m}$. This spectrum distinctly shows the $9.7 \mu \mathrm{m}$ silicate emission feature typical for Orich dust, together with an important infrared excess.

The spectral energy distribution for HD 62623 is shown in Fig. 1 together with the IRAS LRS spectrum. The near UV and optical data can be fitted with a solar composition Kurucz (1979) model atmosphere with $T_{\text {eff }}=9000 \mathrm{~K}$ and $\log g=2$. The Kurucz model overestimates the far UV flux. This may be due to the fact that the star is surrounded by circumstellar dust which becomes optically thick at short wavelengths. We didn't apply an extinction correction to the Kurucz model because we are interested in the near infrared part of the spectral energy distribution curve.

\subsection{High angular observations made at Keck I}

Aperture masking interferometry was performed by placing custom-made plates in front of the Keck I secondary mirror, reducing light from the $10 \mathrm{~m}$ primary mirror to a predefined set of subapertures. The introduction of such a mask creates a series of overlapping interference patterns projected onto the detector array, allowing the Fourier amplitude and phase of each baseline to be recovered without the addition of "redundancy" noise (Baldwin et al. 1986). In the bright source limit, nonredundant aperture masking delivers a maximal signal-to-noise ratio of the closure phases, which is critical for reconstructions of noncentrosymmetric images. For fainter sources, a doubly redundant mask geometry in the shape of an annulus was used (Haniff \& Buscher 1992), balancing the desire for stellar flux with that for limited baseline redundancy. Further engineering and performance details may be found in Tuthill et al. (2000). Raw visibilities were calibrated for the mean telescope-atmosphere transfer function by utilizing nearly contemporaneous observations of point-source stars, under nearly identical conditions. Even so, seeing and other changes make the calibration process notoriously difficult, and mismatches between source and calibrator data introduced the greatest uncertainty into the final results.

HD 62623 was observed in both December of 1997 and April 1999 at Keck I using the Near-Infrared Camera (NIRC), a cryogenically cooled $256 \times 256$ pixel InSb array (Matthews \& Soifer 1994). The observations were performed at 1.24, 1.65, 2.26 and $3.08 \mu \mathrm{m}$ and the visibility curves obtained are shown in Fig. 2. The visibilties plotted have been azimuthally averaged. From Fig. 2 it seems that the star is partially resolved at $1.24,2.26$ and $3.08 \mu \mathrm{m}$ whereas at $1.65 \mu \mathrm{m}$ it is unresolved (as indicated by a flat visibility curve with spatial frequency). A serious note of caution must be expressed over the reliability of the angular sizes determined from the visibility data. The falloff in visibility seen is not large, and at a level which can be comparable to systematic errors which occur due to mismatches of the atmospheric conditions between observations of the star and calibrator. The formal errors from the curve-fitting are always small (usually less than one 
Table 1. A compilation of photometric data of HD 62623 from Rovero \& Riguelet (1994) (Table A), and from Trams et al. (1991) (Table B)

\begin{tabular}{|c|c|c|c|c|c|c|c|c|}
\hline Table A & $\begin{array}{c}\lambda \\
(\mu \mathrm{m})\end{array}$ & $\begin{array}{l}\text { Flux } \\
\text { (mag) }\end{array}$ & $\begin{array}{c}\log F_{\lambda} \\
\left(\mathrm{erg} / \mathrm{cm}^{2} \mu \mathrm{m} \mathrm{s}\right)\end{array}$ & Table B & $\begin{array}{c}\lambda \\
(\mu \mathrm{m})\end{array}$ & $\begin{array}{l}\text { Flux } \\
\text { (mag) }\end{array}$ & (Jy) & $\begin{array}{c}\log F_{\nu} \\
\left(\mathrm{erg} / \mathrm{cm}^{2} \mathrm{~s} \mathrm{~Hz}\right)\end{array}$ \\
\hline$U B V$ & $\begin{array}{l}0.36 \\
0.44 \\
0.55\end{array}$ & $\begin{array}{l}4.05 \\
4.14 \\
3.96\end{array}$ & $\begin{array}{l}-5.79 \\
-5.65 \\
-5.87\end{array}$ & $\begin{array}{c}\text { Wesselius et al. } \\
(1982)\end{array}$ & $\begin{array}{l}0.155 \\
0.180 \\
0.220 \\
0.250\end{array}$ & $\begin{array}{l}5.936 \\
5.430 \\
5.790 \\
5.609\end{array}$ & & $\begin{array}{l}-22.91 \\
-22.58 \\
-22.72 \\
-22.36\end{array}$ \\
\hline $\begin{array}{c}\text { Johnson } \\
\&\end{array}$ & $\begin{array}{l}0.337 \\
0.354\end{array}$ & $\begin{array}{l}4.45 \\
4.32\end{array}$ & $\begin{array}{l}-6.05 \\
-6.04\end{array}$ & & 0.330 & 4.564 & & -21.70 \\
\hline $\begin{array}{l}\text { Mitchell } \\
(1975)\end{array}$ & $\begin{array}{l}0.375 \\
0.403 \\
0.457 \\
0.518 \\
0.583\end{array}$ & $\begin{array}{l}3.89 \\
4.20 \\
4.19 \\
4.05 \\
3.93\end{array}$ & $\begin{array}{l}-5.74 \\
-5.62 \\
-5.73 \\
-5.86 \\
-6.10\end{array}$ & $\begin{array}{c}\text { Thompson et al. } \\
\text { (1978) }\end{array}$ & $\begin{array}{l}0.1565 \\
0.1965 \\
0.2365 \\
0.2740\end{array}$ & & & $\begin{array}{l}-22.84 \\
-22.48 \\
-22.50 \\
-22.07\end{array}$ \\
\hline & $\begin{array}{l}0.636 \\
0.724 \\
0.800 \\
0.858 \\
0.983 \\
1.108\end{array}$ & $\begin{array}{l}3.84 \\
3.73 \\
3.69 \\
3.52 \\
3.51 \\
3.42\end{array}$ & $\begin{array}{l}-6.06 \\
-6.20 \\
-6.34 \\
-6.38 \\
-6.51 \\
-6.65\end{array}$ & 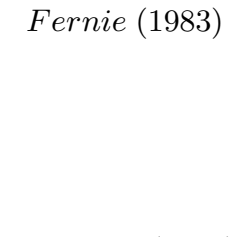 & $\begin{array}{l}0.36 \\
0.44 \\
0.55 \\
0.70 \\
0.90\end{array}$ & $\begin{array}{l}4.05 \\
4.16 \\
3.97 \\
3.70 \\
3.56\end{array}$ & & $\begin{array}{l}-21.35 \\
-21.00 \\
-20.99 \\
-21.02 \\
-21.07\end{array}$ \\
\hline Allen (1973) & $\begin{array}{l}1.60 \\
2.20 \\
3.50\end{array}$ & $\begin{array}{l}3.07 \\
2.33 \\
0.84\end{array}$ & $\begin{array}{l}-7.06 \\
-7.33 \\
-7.47\end{array}$ & Rufener (1988) & $\begin{array}{l}0.3464 \\
0.4015 \\
0.4227 \\
0.4476 \\
0.5395\end{array}$ & $\begin{array}{l}4.81 \\
4.07 \\
3.21 \\
4.72 \\
4.68\end{array}$ & & $\begin{array}{l}-21.56 \\
-21.07 \\
-21.05 \\
-21.03 \\
-21.01\end{array}$ \\
\hline $\begin{array}{l}\text { The et al. } \\
\text { (1986) }\end{array}$ & $\begin{array}{l}1.25 \\
1.65 \\
2.20\end{array}$ & $\begin{array}{l}3.40 \\
3.08 \\
2.36\end{array}$ & $\begin{array}{l}-6.79 \\
-7.10 \\
-7.34\end{array}$ & & $\begin{array}{l}0.5488 \\
0.5807\end{array}$ & $\begin{array}{l}3.96 \\
5.10\end{array}$ & & $\begin{array}{l}-21.01 \\
-21.01\end{array}$ \\
\hline & $\begin{array}{l}3.40 \\
4.80\end{array}$ & $\begin{array}{l}0.83 \\
0.17\end{array}$ & $\begin{array}{l}-7.41 \\
-7.73\end{array}$ & $\begin{array}{c}\text { Trams et al. } \\
\text { (1990) } \\
\text { Koornneef }\end{array}$ & $\begin{array}{l}1.25 \\
1.65 \\
2.25\end{array}$ & $\begin{array}{l}3.26 \\
3.10 \\
2.33\end{array}$ & & $\begin{array}{l}-21.10 \\
-21.21 \\
-21.11\end{array}$ \\
\hline Simon (1976) & 20.0 & -3.00 & -8.94 & $\begin{array}{c}(1983) \\
\text { Epchtein et al. } \\
(1980)\end{array}$ & $\begin{array}{c}3.7 \\
4.8 \\
8.6 \\
10.2 \\
10.7 \\
12.2 \\
18.0\end{array}$ & $\begin{array}{c}0.64 \\
-0.06 \\
-1.53 \\
-2.06 \\
-1.87 \\
-2.7 \\
-3.5\end{array}$ & & $\begin{array}{l}-20.77 \\
-20.79 \\
-20.68 \\
-20.62 \\
-20.62 \\
-20.58 \\
-20.68\end{array}$ \\
\hline & & & & $I R A S$ & $\begin{array}{c}12 . \\
25 . \\
60 . \\
100 .\end{array}$ & & $\begin{array}{l}211.3 \\
114.3 \\
19.05 \\
6.412\end{array}$ & $\begin{array}{l}-20.68 \\
-20.94 \\
-21.72 \\
-22.19\end{array}$ \\
\hline
\end{tabular}

milli-arcsecond). This error is not a realistic measurement of the true uncertainty. The real error of the measurement comes from unknown changes in the atmospheric conditions between the observations of the source and calibrator star. This is not reflected in the error bars on the plots. Thus the only real way to get realistic errors is to repeat measurements a number of times and look at the spread of values we get.

Visibilities have been interpreted in the context of a model consisting of a central hot star surrounded by a circumstellar envelope of gas and dust and at a large radius. Within this picture, the extended $1.24 \mu \mathrm{m}$ emission may originate from light from the central star that is scattered by dust grains. This implies the presence of a population of efficient scatterers at this wavelength. The partially resolved 2.26 and $3.08 \mu \mathrm{m}$ curves may be interpreted as the thermal reradiation from hot silicate dust grains at a temperature of about $1500 \mathrm{~K}$ which is the condensation temperature given for silicate grains (Lamers \& Cassinelli 1999). The presence of these grains is also supported by the low resolution spectrum of IRAS (Fig. 1) showing the emission feature at $9.7 \mu \mathrm{m}$. The most striking effect is coming from the $1.65 \mu \mathrm{m}$ visibility curve (not very far in wavelength from the $1.24 \mu \mathrm{m}$ observations) which seems 

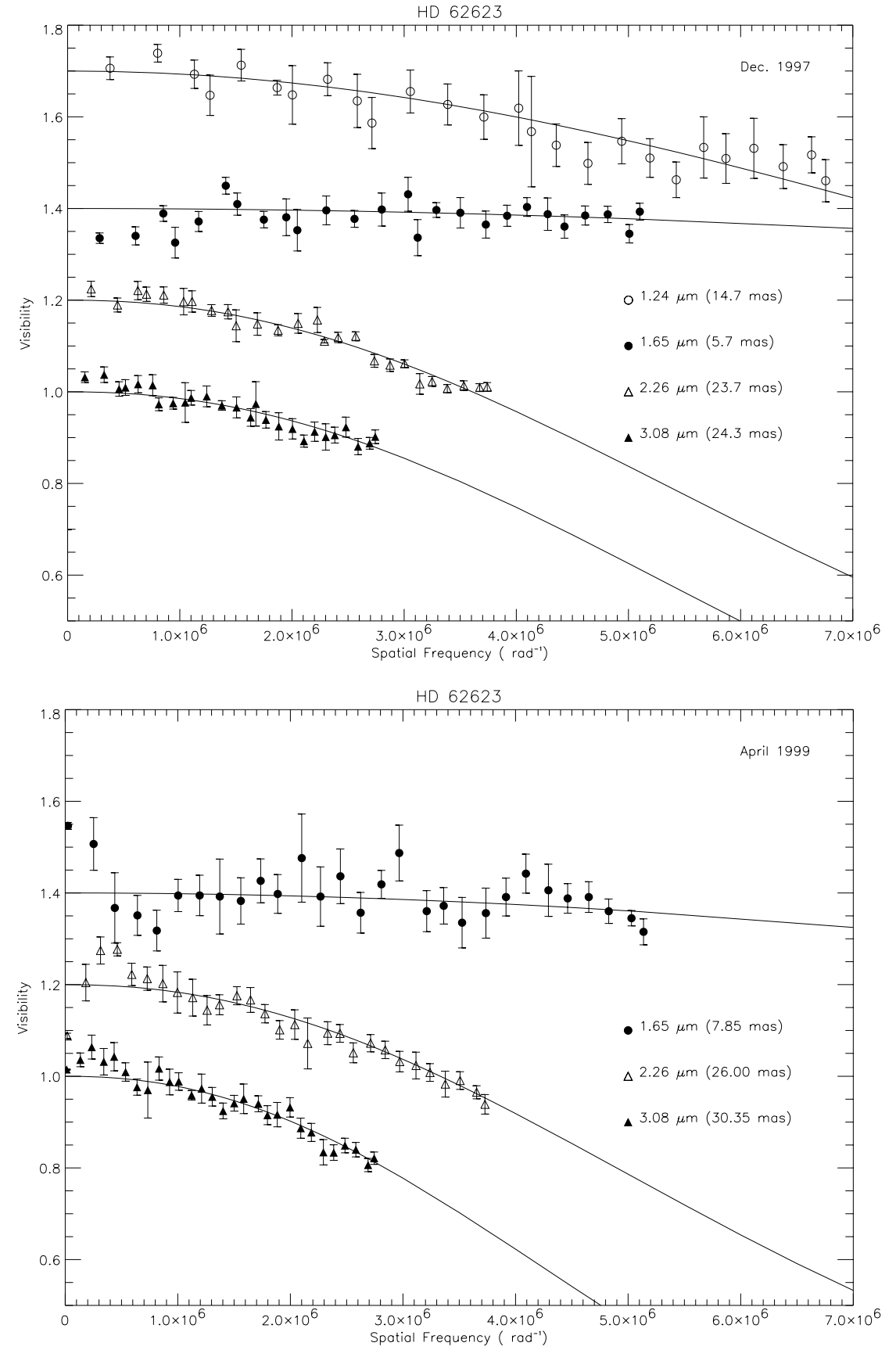

Fig. 2. Visibility curves obtained at the Keck I telescope for the star HD 62623 and plotted as a function of baseline length by performing an azimuthal average. The upper graph corresponds to observations obtained in December 1997 for four wavelengths, while the lower graph corresponds to data obtained in April 1999 for three wavelengths. Constant offsets have been added to the various colors to separate them on the plot. The offset is a visibility of +0.7 for the $1.24 \mu \mathrm{m},+0.4$ for the $1.65 \mu \mathrm{m}+0.2$ for the $2.26 \mu \mathrm{m}$ and 0 for the $3.08 \mu \mathrm{m}$ for the Dec. 97 data. For the April 99, the offset is a visibility of +0.4 for the $1.65 \mu \mathrm{m},+0.2$ for the $2.26 \mu \mathrm{m}$ and 0 for the $3.08 \mu \mathrm{m}$ observations. The lines correspond to Gaussian fits, with the FWHM given in the key of each plot to the right. The formal errors from the curve-fitting are always small (usually less than one milli-arcsecond). This error is not a realistic measurement of the true uncertainty. The real error of the measurement comes from unknown changes in the atmospheric conditions between the observations of the source and calibrator star. This is not reflected in the error bars on the plots. Thus the only real way to get realistic errors is to repeat measurements a number of times and look at the spread of values we get to indicate an unresolved object, a result that was confirmed with the April 1999 observations. This implies that HD 62623 is less extended at this wavelength and that the origin of the $1.65 \mu \mathrm{m}$ emission is therefore mainly photospheric.

\section{Modeling versus observations}

\subsection{The model}

In order to derive a quantitative understanding, we have modeled the circumstellar environment of HD 62623 using a code developed by Lopez et al. (1993, 1995) based on a Monte Carlo method from Lefèvre et al. (1982). This code enabled us to obtain intensity maps in the infrared, after solving the radiative transfer and equilibrium equations. Visibilities extracted from such model maps can be directly compared to our high angular resolution observations.

We assume that the stellar photosphere radiates as a blackbody characterized by its effective temperature $T_{\text {eff }}$ and is surrounded by a spherical dust shell that is responsible for the observed infrared excess. The stellar radiation and the thermal emission from dust are simulated photon by photon. Some fraction of the radiation escapes the shell directly while the remainder interacts with the dust grains. For each interaction with a grain, the radiation is partially absorbed and partially scattered. The new direction of propagation for the photon is then generated by a random process which follows the scattering diagram. The spherical dust shell is bounded by an inner and outer 

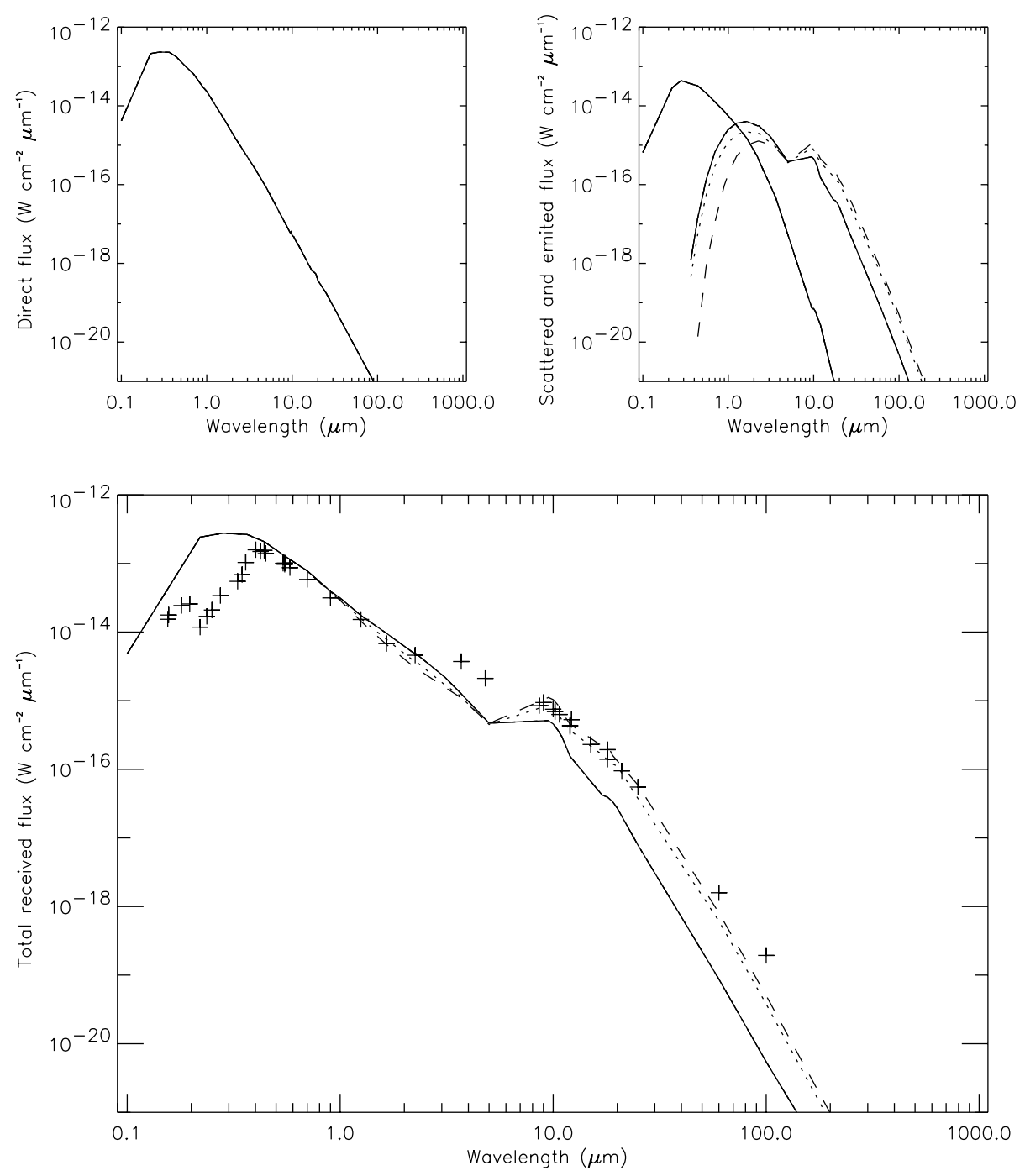

Fig. 3. The spectrum of HD 62623 compared to what is obtained from modeling for a central source embedded in a spherical dust shell. The dashed line represents model $\mathrm{A}$, the dotted line represents model B while the solid line represents model $\mathrm{C}$. The left side upper graph gives the contribution of the source while the right side upper graph gives the contribution of the scattering and of thermal reemission by dust. The lower graph gives the total received flux compared to the observations

radius denoted $R_{\text {int }}$ and $R_{\text {out }}$ expressed in stellar photospheric radius units.

The dust grains are assumed to be of a homogeneous composition and spherical with radius $a$. While a grain size distribution is expected in dust clouds, a unique grain radius is considered here. This means that the model neglects the effects of grains very different from the size we consider. Dust particles are supposed to be composed of silicate as proposed by the low resolution spectrum of IRAS that distinctly shows the $9.7 \mu \mathrm{m}$ emission feature. for the modeling, we used astronomical silicate dust grains from Draine \& Lee (1984). The dust grains number density $N(r)$ is a power law distribution where $r$ is the distance from the center of the star given in stellar photospheric radius units.

The optical depth in the dust shell is defined along the line of sight by:

$\tau_{\text {ext }}(\lambda)=\int_{R_{1}}^{R_{2}} N(r) \pi a^{2} Q_{\text {ext }}(\lambda) \mathrm{d} x$.

Where $a$ is the grain radius. $C_{\text {ext }}=\pi a^{2} Q_{\text {ext }}$ is the extinction cross section, $Q_{\text {ext }}(\lambda)$, the extinction efficiency is obtained from the Mie theory. The extinction, related to the absorption and scattering by $C_{\text {ext }}=C_{\text {abs }}+C_{\text {sca }} \cdot \tau$, is calculated step by step along the direction of propagation until the photon leaves the shell or, as sometimes arises, it encouters the central star.

Thirty wavelengths are used to represent the emergent spectrum from the star and the emergent spectrum from the dust particles. The calculation gives the broad band energy distribution and the grain temperature versus the distance from the central star. Synthetic visibility curves at different wavelengths are created using the synthetic images at any wavelength.

\subsection{Results}

We try here to reproduce or rather to approach the behaviour of the spectrum and of the visibility curves by empirically adjusting our model parameters.

The best fit of the spectral energy distribution is obtained for $T_{\text {eff }}=10000 \mathrm{~K}, \tau_{\text {ext }}(\lambda=1 \mu \mathrm{m})=0.30$, a grain radius, $a$, varying from $0.5 \mu \mathrm{m}$ to $1.5 \mu \mathrm{m}, R_{\text {int }}=$ $35 R_{*}$ and $R_{\text {out }}=5000 R_{*}$ with a radial dust density distribution of $r^{-1.3}$ as proposed by Plets et al. (1995). (model A, dashed line in Fig. 3). The deviation from the 

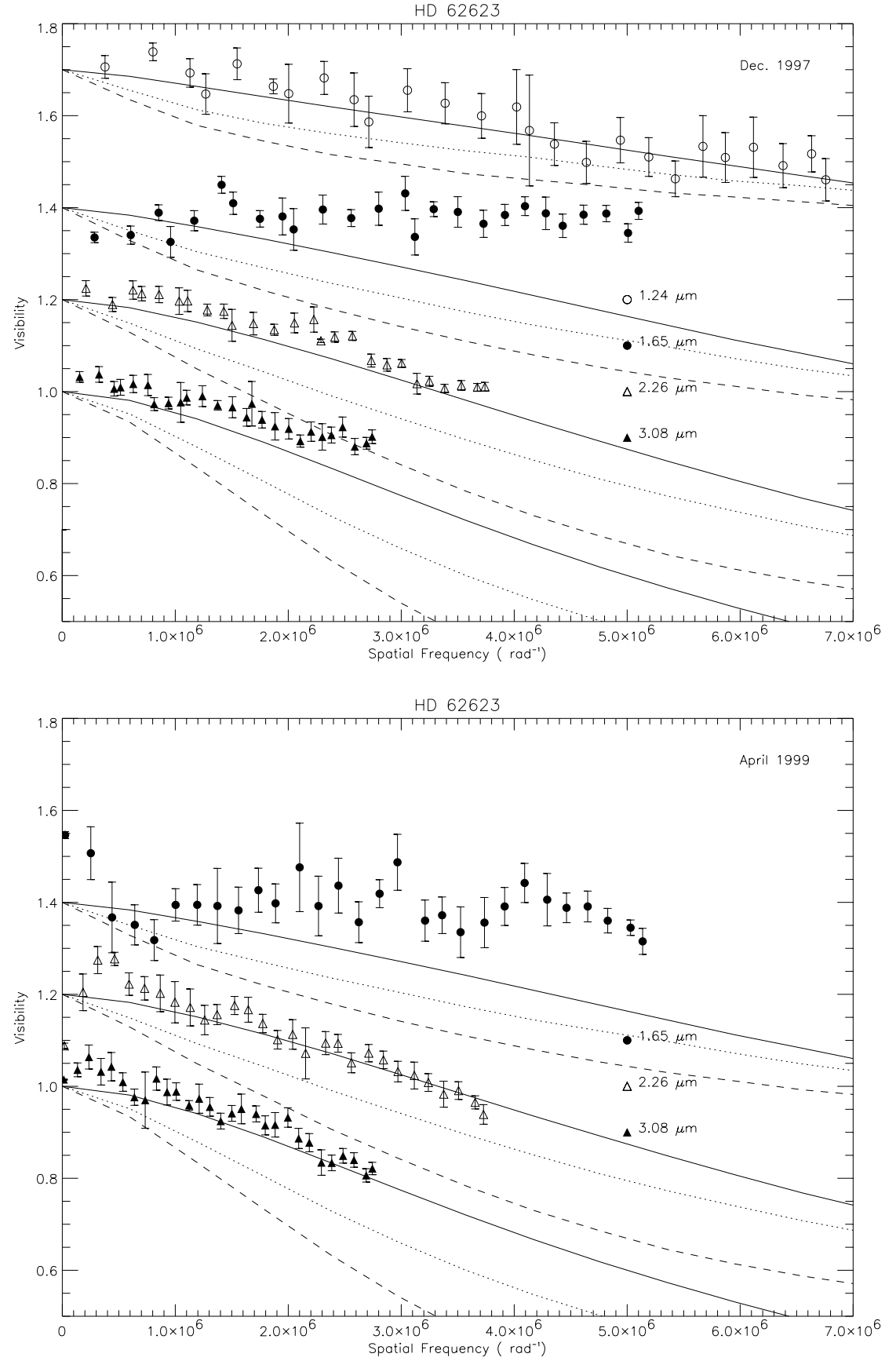

Fig. 4. The fit of the visibility curves obtained at the Keck I telescope for the star HD 62623. The upper graph corresponds to the December 1997 observations; the offset is a visibility of +0.7 for the $1.24 \mu \mathrm{m},+0.4$ for the $1.65 \mu \mathrm{m}$, +0.2 for the $2.26 \mu \mathrm{m}$ and 0 for the $3.08 \mu \mathrm{m}$. The lower graph corresponds to April 1999 observations where the offsets are respectively $+0.4,+0.2$ and 0 for the $1.65 \mu \mathrm{m}, 2.26 \mu \mathrm{m}$ and $3.08 \mu \mathrm{m}$ observations. The dashed lines are the theoretical visibility curve corresponding to the simulated images at different wavelength for model A the dotted lines for model B while the solid lines correspond to model $\mathrm{C}$
UV observations (IUE spectra) may be due to the use of a blackbody for representing the central star. The theoretical visibility curves calculated for the parameters of model $\mathrm{A}$ are represented by the dashed line in Fig. 4 and show an important deviation from the observed visibilities in the near infrared. This shows the importance of including the interferometric results in the constraints of the modeling since the spectrum alone is insufficient to provide meaningful constraints.

The spectral energy distribution may be fitted also by another set of paramaters (model B in Table 2) that gives less resolved visibilities due to a smaller inner radius of the dust shell. The results for model B are shown by the dotted line in Figs. 3 and 4.
A third model $(\mathrm{C})$ is developed with the same parameters except for the density distribution law that decreases more rapidly with a greater power (a density falling as $\left.r^{-2}\right)$. This model reproduces less faithfully the observed flux especially for the far infrared excess but it gives theoretical visibility curves that are in better agreement with the interferometric observations. The results are shown by the solid line in Figs. 3 and 4 . All the models that fit well the $L$ and $M$ bands were not able to reproduce an acceptable fit of the visibilities observed under the assumptions of our model. The combination of inner radius, grain size and optical depth that fits both the photometric and the interferometric data is somehow unique within an interval of $0.4 \mu \mathrm{m}$ for the grain size, $4 R_{*}$ for the inner 
radius and 0.1 for the optical depth. The parameters for the three models (model A, model $\mathrm{B}$ and model $\mathrm{C}$ ) are represented in Table 2. Models B and $\mathrm{C}$ have the advantage that the smaller inner radius for the dust shell agrees with the less resolved visibilities observed. The angular radius $\left(R_{*} / d\right)$ of the star is found to be $\alpha_{*}=1.510^{-9}$ radians consistent with the amount of the received flux and with the value given by Rovero et al. (1994), i.e. $1.4610^{-9}<\alpha_{*}<1.7610^{-9}$.

Table 2. Parameters used to fit the spectral energy distribution of HD 62623. $a_{\text {grain }}$ is the grain size in $\mu \mathrm{m}, R_{\text {int }}$ and $R_{\text {ext }}$ are the inner and outer radius of the shell in units of stellar radii, $\tau$ is the optical depth at $1 \mu \mathrm{m}$ and $\alpha_{*}=R_{*} / d_{*}$ in $\operatorname{rad}$

\begin{tabular}{llll}
\hline Parameters & model A & model B & model C \\
\hline$T_{\text {eff }}$ & 10000 & 10000 & 10000 \\
$\alpha_{*}$ & $1.510^{-9}$ & $1.510^{-9}$ & $1.510^{-9}$ \\
$a_{\text {grain }}$ & 0.75 & 0.75 & 0.75 \\
$R_{\text {int }}$ & 35 & 15 & 15 \\
$R_{\text {ext }}$ & 5000 & 5000 & 5000 \\
$n(r)$ & $\sim r^{-1.3}$ & $\sim r^{-1.3}$ & $\sim r^{-2}$ \\
$\tau(\lambda=1 \mu \mathrm{m})$ & 0.30 & 0.30 & 0.30 \\
\hline
\end{tabular}

We also tested the effect of the grain size on the spectral energy distribution and on the theoretical visibility curves. Smaller grains at a constant radius produce less resolved visibility curves, approaching the observations, but lowering the infrared emission resulting in a bad fit to the photometric data. On the other hand bigger grains generate more emission, and therefore are better able to fit photometric data but not the interferometric observations. We tested the grain size independently of the inner radius to understand its effect on the theoretical visibility curves.

A problem remains for the $1.65 \mu \mathrm{m}$ visibility curves that show an object that seems to be observationally unresolved, a finding found to be consistent between both epochs of data presented. It is not inconceiveable that the envelope may be more transparent at this wavelength and that the received light may orginate from the underlying photosphere or very close to it. We failed to reproduce the $1.65 \mu \mathrm{m}$ visibility curve (unresolved object) while having a more resolved object at $1.24 \mu \mathrm{m}$ under the assumptions of the model. However, we hasten to reiterate that in the barely-resolved regime, calibration errors might account for some such anomalies.

\section{Conclusion}

The first high angular resolution observations of HD 62623 indicate that this peculiar star is resolved at three nearIR wavelengths whereas at $1.65 \mu \mathrm{m}$ it is apparently unresolved. This finding should be regarded as tentative until instruments with longer baselines are able to more fully resolve the visibility function. A simple model consisting of a central source and a spherical dust shell is able to reproduce the observed spectral energy distribution reasonably well from the visible to the far IR (but not the UV). We have shown that including interferometric results in the modeling of the circumstellar envelopes puts some important additional constraints. Such a model was able to reproduce the near-IR inteferometric observations obtained at the Keck I telescope using the aperture masking interferometry for three wavelengths $1.24 \mu \mathrm{m}, 2.26 \mu \mathrm{m}$ and $3.08 \mu \mathrm{m}$ but not for the $1.65 \mu \mathrm{m}$ visibility curve that shows an unresolved object at this wavelength.

Mis-calibration of the visibility function is the most likely origin for the behavior of the $1.65 \mu \mathrm{m}$ data, although it is also possible that the source is less extended at this wavelength. If the latter is true, we expected to reproduce this behaviour using a model involving scattering and emission properties which varied with $\lambda$. We failed to produce significant $1.65 \mu \mathrm{m}$ deviation in our models. An alternate explanation involves the presence of hot circumstellar gas that emits as an extended source at $1.24 \mu \mathrm{m}$ and that is masking the central star, an effect already predicted by Stee 1998 for B[e] stars with mass loss larger than $10^{-5} M_{\odot} \mathrm{yr}^{-1}$. At $1.65 \mu \mathrm{m}$ the hot circumstellar gas emission may be less opaque and thus most of the flux at this wavelength originates from the central unresolved star. Even if you have a more complicated geometry, like a disk, producing an inclination angle dependence of the source (star + dust) visibility you still need a physical effect which strongly varies with the wavelength.

In conclusion, models have been constructed which account for the presence of hot dust in a spherical shell around HD 62623. Despite the simple approach, this was found to be a good approximation even though it was not able to explain the visibility curves at all the wavelengths observed. In the future, we hope to present a more accurate model with a non-spherical circumstellar envelope which includes both gas and dust in order to test a more realistic approximation to the environment of HD 62623.

Acknowledgements. Interferometry research at U. C. Berkeley was sponsored by the National Science Foundation (AST 9731625) and by the National Aeronautics and Space Administration. Some of the measurements here in were obtained at the W. M. Keck Observatory and made possible by the generous support of the W. M. Keck Foundation and operated as a scientific partnership among the California Institute of Technology, the University of California, and NASA. The useful remarks of the referee L. B. F. M. Waters are greatly acknowledged by the authors.

\section{References}

Allen, D. A. 1973, MNRAS, 161, 145

Baldwin, J. E., Haniff, C. A., Mackay, C. D., \& Warnier, P. J. 1986, Nature, 320, 595

Draine, B. T., \& Lee, H. M. 1984, ApJ, 285, 89

Epchtein, N., Guibert, J., Nguyen-Quang-Rieu, Turon, P., \& Wamsteker, W. 1980, A\&A, 85, L1

Fernie, J. D. 1983, ApJS, 52, 7

Haniff, C. A., \& Buscher, D. F. 1992, J. Opt. Soc. Am., 9, 203

Hoffleit, D., \& Jaschek, C. 1982, The Bright Star Catalogue, Yale University Observatory, New Haven, USA 
IRAS, Science Team 1986, A\&AS, 65, 617

Johnson, H. L., \& Mitchell, R. I. 1975, RMxAA, 1, 299

Koornneef, J. 1982, A\&A, 128, 84

Kurucz, R. L. 1979, ApJS, 40, 1

Kwok, S., Volk, K., \& Bidelman, W. P. 1997, ApJS, 112, 557

Lambert, D. L., Hinkle, K. H., \& Luck, R. E. 1988, ApJ, 333, 917

Lamers, H. J. G. L. M., \& Cassinelli, J. P. 1999, Introduction to stellar winds (Cambridge University Press)

Lefèvre, J., Bergeat, J., \& Daniel, J. Y. 1982, A\&A, 114, 341

Lopez, B., Perrier, C., Mékarnia, D., Lefèvre, J., \& Gay, J. 1993, A\&A, 270, 462

Lopez, B., Mékarnia, D., \& Lefèvre, J. 1995, A\&A, 296, 752

Lodén, L. O., \& Sundman, A. 1995, A\&AS, 112, 9

Matthews, K., \& Soifer, B. T. 1994, ExA, 3, 77

Monnier, J. D., Tuthill, P. G., Lopez, B., et al. 1999, ApJ, 512, 351

Plets, H., Waelkens, C., \& Trams, N. R. 1995, A\&A, 293, 263

Rovero, A. C., \& Ringuelet, A. E. 1994, A\&A, 266, 203

Rufener, F. 1988, Catalogue of stars measured in the Geneva Observatory photometric system, Fourth Edition, Obs. de Genève
Simon, T. 1976, AJ, 81, 764

Stee, Ph. 1998, A\&A, 336, 980

The, P. S., Wesselius, P. R., \& Janssen, I. M. H. H. 1986, A\&AS, 66, 63

Thompson, G. I., Nandy, K., Jamar, C., et al. 1978, Catalogue of Stellar Ultraviolet Fluxes

Trams, N. R., Waters, L. B. F. M., Lamers, H. J. G. L. M., et al. 1991, A\&AS, 87, 361

Trams, N. R., van der Veen, W. E. C. J., Waelkens, C., Waters L. B. F. M., \& Lamers H. J. G. L. M. 1990, A\&A, 233, 153

Trams, N. R., Lamers, H. J. G. L. M., Waters, L. B. F. M., Waelkens, C., \& Groth, H. G. 1990, in From Miras to Planetary Nebulae: Which Path For Stellar Evolution?, ed. M. O. Mennessier, \& A. Omont (Éditions Frontières, France), 542

Trams, N. R., Waters, L. B. F. M., Waelkens, C., Lamers, H. J. G. L. M., \& van der Veen, W. E. C. J. 1989, A\&A, 218, L1

Tuthill, P. G., Monnier, J. D., Danchi, W. C., Wishnow, E. H. \& Haniff, C. A. 2000, PASP, 112, 555

Wesselius, P. R., Van Duinen, R. J., de Jonge, A. R. W., et al. 1982, A\&AS, 49, 427 